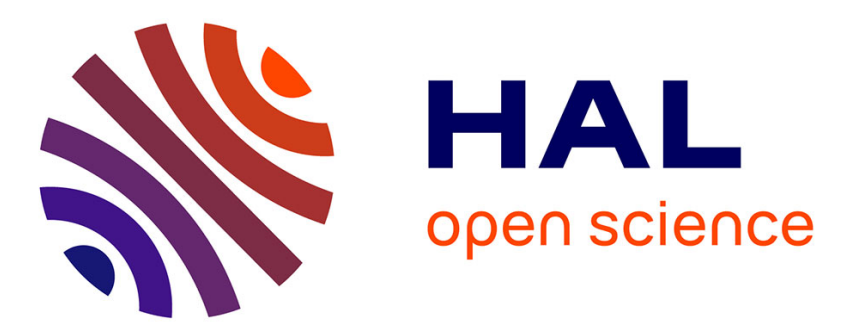

\title{
Effects of structural and functional cerebellar lesions on sensorimotor adaptation of saccades
}

M. Panouillères, N. Alahyane, C. Urquizar, R. Salemme, N. Nighoghossian, B. Gaymard, C. Tilikete, D. Pelisson

\section{- To cite this version:}

M. Panouillères, N. Alahyane, C. Urquizar, R. Salemme, N. Nighoghossian, et al.. Effects of structural and functional cerebellar lesions on sensorimotor adaptation of saccades. Experimental Brain Research, 2013, 231 (1), pp.1-11. 10.1007/s00221-013-3662-6 . hal-03131168

\section{HAL Id: hal-03131168 \\ https://hal.science/hal-03131168}

Submitted on 4 Feb 2021

HAL is a multi-disciplinary open access archive for the deposit and dissemination of scientific research documents, whether they are published or not. The documents may come from teaching and research institutions in France or abroad, or from public or private research centers.
L'archive ouverte pluridisciplinaire HAL, est destinée au dépôt et à la diffusion de documents scientifiques de niveau recherche, publiés ou non, émanant des établissements d'enseignement et de recherche français ou étrangers, des laboratoires publics ou privés.

\section{(c)(1)}

Distributed under a Creative Commons Attribution| 4.0 International License 


\title{
Effects of structural and functional cerebellar lesions on sensorimotor adaptation of saccades
}

\author{
M. Panouillères $\cdot$ N. Alahyane $\cdot$ C. Urquizar $\cdot$ \\ R. Salemme $\cdot$ N. Nighoghossian $\cdot$ B. Gaymard \\ C. Tilikete $\cdot$ D. Pélisson
}

Received: 13 December 2012 / Accepted: 28 July 2013 / Published online: 21 August 2013

(C) Springer-Verlag Berlin Heidelberg 2013

\begin{abstract}
The cerebellum is critically involved in the adaptation mechanisms that maintain the accuracy of goaldirected acts such as saccadic eye movements. Two categories of saccades, each relying on different adaptation mechanisms, are defined: reactive (externally triggered) saccades and voluntary (internally triggered) saccades. The contribution of the medio-posterior part of the cerebellum to reactive saccades adaptation has been clearly demonstrated, but the evidence that other parts of the cerebellum are also involved is limited. Moreover, the cerebellar substrates of voluntary saccades adaptation have only been marginally investigated. Here, we addressed these two questions by investigating the adaptive capabilities of patients with cerebellar or pre-cerebellar stroke. We recruited three groups of patients presenting focal lesions located, respectively,
\end{abstract}

Electronic supplementary material The online version of this article (doi:10.1007/s00221-013-3662-6) contains supplementary material, which is available to authorized users.

M. Panouillères $\cdot$ C. Urquizar $\cdot$ R. Salemme $\cdot$ C. Tilikete · D. Pélisson

INSERM U1028, CNRS UMR5292, ImpAct Team, Lyon

Neuroscience Research Center, 69000 Lyon, France

M. Panouillères · C. Urquizar · R. Salemme $\cdot$ N. Nighoghossian ·

C. Tilikete $\cdot$ D. Pélisson

University Lyon 1, 69000 Lyon, France

M. Panouillères $(\square)$

INSERM U1028, CNRS UMR5292, ImpAct Team, Lyon Neuroscience Research Center, 16 avenue du doyen Lépine, 69676 Bron Cedex, France

e-mail: muriel.panouilleres@inserm.fr

N. Alahyane

Laboratoire Vision Action Cognition, IUPDP Université Paris

Descartes, 92774 Boulogne-Billancourt Cedex, France in the supero-anterior cerebellum, the infero-posterior cerebellum and the lateral medulla (leading to a Wallenberg syndrome including motor dysfunctions similar to those resulting from lesion of the medio-posterior cerebellum). Adaptations of reactive saccades and of voluntary saccades were tested during separate sessions in all patients and in a group of healthy participants. The functional lesion of the medio-posterior cerebellum in Wallenberg syndrome strongly impaired the adaptation of both reactive and voluntary saccades. In contrast, patients with lesion in the supero-anterior part of the cerebellum presented a specific adaptation deficit of voluntary saccades. Finally, patients with an infero-posterior cerebellar lesion showed mild adaptation deficits. We conclude that the medio-posterior cerebellum is critical for the adaptation of both saccade categories, whereas the supero-anterior cerebellum is specifically involved in the adaptation of voluntary saccades.

N. Nighoghossian

Hospices Civils de Lyon, Unité Cérébrovasculaire, Hôpital Neurologique, 69677 Bron, France

B. Gaymard

INSERM UMRS 975, CNRS 7225, Pierre et Marie Curie

Paris-6 University, Paris, France

B. Gaymard

Assistance Publique Hôpitaux de Paris (AP-HP), Fédération

of Clinical Neurophysiology, Pitié-Salpêtrière Hospital,

Paris, France

C. Tilikete

Hospices Civils de Lyon, Unité de Neuro-ophtalmologie and Service de Neurologie D, Hôpital Neurologique,

69677 Bron, France 
Keywords Cerebellum $\cdot$ Wallenberg $\cdot$ Saccades . Adaptation

\section{Introduction}

The cerebellum is a key structure in the control and sensorimotor adaptation mechanisms of saccadic eye movements (Glickstein et al. 2011). Saccades triggered by exogenous cues, like the sudden appearance of a new object, belong to the category of reactive saccades. But to explore our visual environment, we most frequently generate scanning saccades which are triggered according to internal goals and which belong to the voluntary saccades category. The different properties of these two types of saccades are linked to anatomo-functional specificities of the oculomotor areas of the cerebral cortex (Johnston and Everling 2008; Muri and Nyffeler 2008). At the level of the cerebellum instead, evidence for such specialisation is scarce (Schraa-Tam et al. 2009) and contradicted by recent monkey data showing undifferentiated neuronal activities in the medio-posterior cerebellum for the generation of these two saccade types (Kojima et al. 2010). Regarding the adaptation mechanisms maintaining saccade accuracy (so-called saccadic adaptation), several behavioural studies have highlighted that they differ for reactive and voluntary saccades, but they did not test the neural substrates underlying this difference (Alahyane et al. 2007; Collins and Dore-Mazars 2006; Cotti et al. 2007; Cotti et al. 2009; Deubel 1995; Erkelens and Hulleman 1993; Fujita et al. 2002; Zimmermann and Lappe 2009).

Concerning adaptation mechanisms that allow decreasing the amplitude (adaptive shortening) of reactive saccades, a major contribution of the medio-posterior cerebellum has been demonstrated (oculomotor vermis composed of lobules VI-VII and caudal part of the fastigial nucleus: Hopp and Fuchs 2004; Iwamoto and Kaku 2010; Pelisson et al. 2010; Prsa and Thier 2011; Tian et al. 2009). This has initially been based on lesion studies in animals (Barash et al. 1999; Optican and Robinson 1980; Takagi et al. 1998) and then in human patients with cerebellar lesions affecting the vermis (Golla et al. 2008) as well as in patients with a neurodegenerative disease primarily affecting the Purkinje cells, particularly in the vermis (Xu-Wilson et al. 2009). Although induced by a lesion outside the cerebellum, in the lateral medulla, Wallenberg syndrome is a good model of dysfunction of the medio-posterior cerebellum in humans. Indeed, by interrupting olivo-cerebellar fibres travelling in the inferior cerebellar peduncle, the lesion yields functional deficits such as saccadic lateropulsion that are similar to those resulting from damage of the fastigial nucleus (Helmchen et al. 1994; Straube et al. 1994; Tilikete et al. 2006). Unfortunately, previous studies concerning the adaptive capabilities of Wallenberg patients provided contradictory results (Choi et al. 2008; Waespe and Baumgartner 1992). Despite this controversy, the role of the medio-posterior cerebellum in reactive saccades adaptation has been confirmed by other approaches (Desmurget et al. 1998; Jenkinson and Miall 2010). To our knowledge, no previous studies tested the involvement of the medio-posterior cerebellum in voluntary saccades adaptation. Moreover, very little is known regarding the involvement of the cerebellar hemispheres in either reactive saccades or voluntary saccades adaptation. A recent transcranial magnetic stimulation (TMS) study disclosed a contribution of the hemispheric lobule Crus I in the adaptation of reactive saccades (Panouilleres et al. 2012a). A role of the cerebellar hemisphere was also suggested by deficits of adaptation of reactive saccades (Alahyane et al. 2008b; Choi et al. 2008; Golla et al. 2008; Straube et al. 2001) or of voluntary saccades (Alahyane et al. 2008b) in patients with lesions involving both the medial and lateral parts of the cerebellum. A recent study, using functional neuroimaging, investigated the brain areas underlying adaptation of both categories of saccades (Gerardin et al. 2012). Besides the newly demonstrated involvement of the cerebral cortex, the authors reported that hemispheric lobules VIIb-VIIIa of the cerebellum are involved in the adaptation of both saccade types.

The aim of the present study was to investigate the cerebellar substrates of saccadic adaptation in human. We focused on the adaptive shortening mechanisms because, although the most studied behaviourally, their neural bases remain largely unknown. We searched for adaptation deficits resulting from stroke lesions in cerebellar and Wallenberg patients and looked for possible dissociations between reactive and voluntary saccades according to the affected territories.

\section{Methods}

Standard protocol approval, registrations and patients consent

Experimental procedures were approved by the national French committees on human experimentation (CPPRB Lyon B, agreement 2003-036B and DGS agreement 2003/0506). A written informed consent was obtained from all patients and all healthy subjects.

\section{Subjects}

From 2005, we prospectively included 18 patients with isolated focal cerebellar or medullary stroke. Four patients were excluded because of difficulty in understanding and/or in performing the oculomotor tasks. The clinical features 
Table 1 Clinical data of the 14 patients

\begin{tabular}{|c|c|c|c|c|c|c|c|c|c|c|c|c|c|}
\hline \multirow[t]{2}{*}{ Patients } & \multirow[t]{2}{*}{ Sex } & \multirow[t]{2}{*}{ Age } & \multirow[t]{2}{*}{ Delay } & \multirow[t]{2}{*}{ Initial clinical manifestations } & \multicolumn{6}{|c|}{ BARS (at time of examination) } & \multirow{2}{*}{$\begin{array}{l}\text { Type } \\
\text { of stroke }\end{array}$} & \multirow[t]{2}{*}{ Territory } & \multirow[t]{2}{*}{ Side } \\
\hline & & & & & $\mathrm{I}$ & II & III & IV & $\mathrm{V}$ & Total & & & \\
\hline $\mathrm{P} 1$ & M & 58 & $9 \mathrm{~d}$ & $\begin{array}{l}\text { Static cerebellar ataxia, right Horner sign, } \\
\text { left spino-thalamic deficit, right saccadic } \\
\text { lateropulsion, left-beating head-shaking } \\
\text { nystagmus }\end{array}$ & 1 & 0 & 0 & 0 & 1 & 2 & Isch & IPICA & Right \\
\hline $\mathrm{P} 2$ & M & 51 & $5 \mathrm{~m}$ & Vertigo and right-beating nystagmus & 0 & 0 & 0 & 0 & 0 & 0 & Isch & $1 \& \mathrm{mPICA}$ & Right \\
\hline P3 & M & 41 & $25 \mathrm{~m}$ & Vertigo and right-beating nystagmus & 0 & 0 & 0 & 0 & 1 & 1 & Isch & mPICA & Left \\
\hline P4 & M & 63 & $27 \mathrm{~d}$ & $\begin{array}{l}\text { Vertigo, right Horner sign, left spino- } \\
\text { thalamic deficit }\end{array}$ & 0 & 0 & 2 & 0 & 1 & 3 & Isch & mPICA & Right \\
\hline P5 & M & 61 & $4 \mathrm{~m}$ & $\begin{array}{l}\text { Vertigo, left-beating nystagmus, right } \\
\text { kinetic cerebellar syndrome, dysarthria, } \\
\text { left spino-thalamic deficit }\end{array}$ & 3 & 2 & 0 & 0 & 1 & 6 & Isch & mPICA & Right \\
\hline P6 & M & 40 & $10 \mathrm{~d}$ & $\begin{array}{l}\text { Right kinetic cerebellar syndrome, right- } \\
\text { beating nystagmus, right-sided tinnitus }\end{array}$ & 2 & 2 & 2 & 0 & 1 & 7 & Isch & 1SCA & Right \\
\hline P7 & M & 39 & $1.5 \mathrm{~m}$ & Dysarthria & 0 & 0 & 0 & 2 & 0 & 2 & Isch & ISCA & Right \\
\hline P8 & M & 56 & $2 \mathrm{~m}$ & $\begin{array}{l}\text { Ataxia, nausea, right kinetic cerebellar } \\
\text { syndrome, dysarthria }\end{array}$ & 1 & 1 & 1 & 1 & 0 & 4 & Isch & $\begin{array}{l}\text { 1SCA and } \\
\text { AICA }\end{array}$ & Right \\
\hline P9 & M & 50 & $1 \mathrm{~m}$ & Ataxia, right kinetic cerebellar syndrome & 3 & 2 & 2 & 0 & 0 & 7 & Hem & "mSCA" & Right \\
\hline $\mathrm{P} 10$ & $\mathrm{~F}$ & 38 & $18 \mathrm{~m}$ & $\begin{array}{l}\text { Paraesthesia, vertigo, ataxia, left spino- } \\
\text { thalamic deficit, right kinetic cerebellar } \\
\text { syndrome, torsional nystagmus }\end{array}$ & 3 & 2 & 0 & 0 & 1 & 6 & Isch & Medulla & Right \\
\hline P11 & M & 49 & $2 \mathrm{~m}$ & $\begin{array}{l}\text { Ataxia, hiccup, right spino-thalamic } \\
\text { deficit, left kinetic cerebellar syndrome, } \\
\text { left Horner sign, left skew deviation, } \\
\text { torsional nystagmus }\end{array}$ & 2 & 0 & 0 & 0 & 1 & 3 & Isch & Medulla & Left \\
\hline P12 & $\mathrm{F}$ & 38 & $2 \mathrm{~m}$ & $\begin{array}{l}\text { Left axial lateropulsion, swallowing } \\
\text { and phonation deficit, left peri-ocular } \\
\text { pain, right spino-thalamic deficit, left } \\
\text { hemifacial sensory deficit, right-beating } \\
\text { nystagmus }\end{array}$ & 1 & 0 & 0 & 0 & 1 & 2 & Isch & Medulla & Left \\
\hline P13 & M & 58 & $9 \mathrm{~m}$ & $\begin{array}{l}\text { Vertigo, left-beating nystagmus, left } \\
\text { Horner sign, left central facial palsy, } \\
\text { right spino-thalamic deficit, swallowing } \\
\text { and phonation deficit, left skew deviation }\end{array}$ & 2 & 2 & 0 & 1 & 1 & 6 & Isch & Medulla & Left \\
\hline P14 & $\mathrm{F}$ & 36 & $28 \mathrm{~m}$ & $\begin{array}{l}\text { Vertigo, right-beating nystagmus, right } \\
\text { hemiparesia, left spino-thalamic deficit, } \\
\text { right Horner sign, right saccadic latero- } \\
\text { pulsion, right skew deviation }\end{array}$ & 3 & 0 & 0 & 0 & 2 & 5 & Isch & Medulla & Right \\
\hline
\end{tabular}

$M$ male, $F$ female, $d$ day, $m$ month, PICA postero-inferior cerebellar artery, $S C A$ superior cerebellar artery, $l$ lateral branch, $m$ medial branch. We used the thirty-point brief ataxia rating scale (BARS) (Schmahmann et al. 2009) to assess ataxia or cerebellar signs at time of examination. This test translates the classical symptomatology of ataxia into 5 semi-quantitative scores (I, postural and stance disturbances; II, inferior limb movements disturbances; III, superior limb movements disturbances; IV, speech disorders; IV, oculomotor disorders)

of the 14 remaining patients are summarised in Table 1. MRI showed a stroke at the level of the postero-inferior cerebellar artery (PICA) territory in 5 patients (P1-P5, PICA group), of the superior cerebellar artery (SCA) and/or of the antero-inferior cerebellar artery (AICA) in 4 patients (P6-P9, SCA-AICA group) and in the medulla (Wallenberg) in 5 patients (P10-P14, Wallenberg group). The vermis of SCA-AICA and PICA patients was completely spared in four patients and only partly affected in the five remaining patients. Data of patients P5 and P6 have been reported elsewhere (Alahyane et al. 2008b), as well as the data of 6 healthy adults (age 22-48 years old), with no history of neurological disease, who served as controls (Alahyane et al. 2007).

Experimental set-up and design

Participants sat in front of a computer screen (vertical refresh rate: $140 \mathrm{~Hz}$-visual angle: $30^{\circ} \times 40^{\circ}$ ) located at $57 \mathrm{~cm}$, their head being restrained with chin and cheekbone rests. Presentation of stimuli was controlled by a VSG system (Visual Stimuli Generation-CRS Cambridge, UK), 
and subjects were instructed to carefully follow visual targets (black circles of $6 \mathrm{~mm}$ diameter on a grey background). Eye movements were binocularly recorded at $250 \mathrm{~Hz}$ with a helmet-mounted infrared eye tracker (Eyelink 2, SR-Research, Canada).

The paradigm used in the present study is identical to the one reported in previous articles (Alahyane et al. 2007; Alahyane et al. 2008b). Details of the protocol are described in supplementary material. Reactive saccades and scanning voluntary saccades were tested in two separate sessions, with a counterbalanced order between subjects. Each session was composed of 3 phases: pre-adaptation (24 saccades), adaptation (240 saccades), post-adaptation (24 saccades). During the adaptation phase, we used the double-step target paradigm to adaptively shorten saccade amplitude (McLaughlin 1967): at the beginning of each primary horizontal saccade (when eye velocity reached a $70-110 \%$ sec threshold), the visual stimulus (one target for the reactive saccade protocol and the set of four targets and fixation point for the voluntary saccade protocol) was displaced in the direction opposite to the saccade (Fig. 1, top panels, adaptation). This intra-saccadic visual perturbation produced an error between the eyes landing position and the target location which, when repeated over trials, led to a progressive decrease in saccade amplitude. Rightward and leftward saccades were simultaneously adapted by presenting the target at $\pm 8^{\circ}$ pseudo-randomly in the same sessions. The intra-saccadic visual perturbation corresponded to $25 \%$ of initial target eccentricity for the first three adaptation blocks $(3 \times 48$ saccades: a25, b25, c25) and to $40 \%$ for the last two blocks $(2 \times 48$ saccades: d40, e40), respectively. Paradigms based on a similar increase in the size of intra-saccadic target jump were successfully used in the past (Alahyane and Pelisson 2005; Alahyane et al. 2007; Alahyane et al. 2008b; Panouilleres et al. 2011; Panouilleres et al. 2012b; Panouilleres et al. 2013; Wong and Shelhamer 2011; Zimmermann and Lappe 2009). We decided to use this strategy of increasing the size of the intra-saccadic target step in our patients in order to maximise the amount of saccadic adaptation. Finally, short breaks were interleaved between blocks of trials to avoid the development of excessive fatigue in the participants. However, because there were only four breaks during the adaptation phase and because their duration was not constant between subjects (from $30 \mathrm{~s}$ to 2-3 $\mathrm{min}$ ), we did not attempt to study the forgetting of adaptation which might occur during breaks (Ethier et al. 2008; Xu-Wilson et al. 2009).

In the pre-adaptation and post-adaptation phases, subjects performed one block of 24 saccades (12 in each direction) directed towards targets presented at $\pm 8^{\circ}$ relative to the centre of the screen (Fig. 1, bottom panels, pre- and post-adaptation). Instead of an intra-saccadic step, the visual stimulus disappeared at the beginning of the primary
Reactive saccades Voluntary saccades

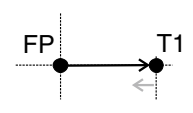

Adaptation
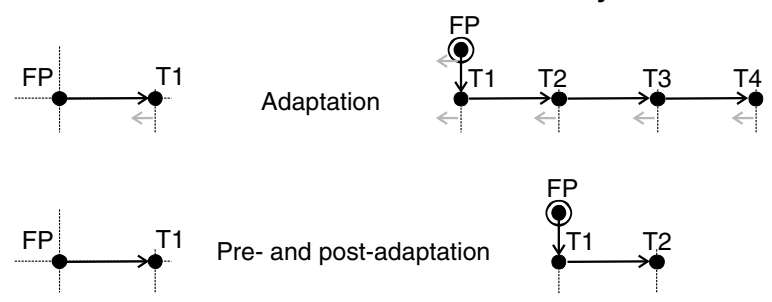

Fig. 1 Protocols for the adaptation (top row) and for the pre- and post-adaptation phases (bottom row). Reactive saccades were triggered by the sudden appearance of a target (T1) at $8^{\circ}$ (in this example, on the right), simultaneously with the disappearance of the fixation point (FP). During an adaptation trial, the initiation of the reactive saccade (black arrow) was responsible for the intra-saccadic jump (short grey arrow) of the target. During pre- and post-adaptation trials, T1 disappeared at saccade onset. In the adaptation trials for the voluntary saccade, the visual stimulus consisted in the fixation point (FP) and 4 targets, all presented simultaneously. At the disappearance of the circle surrounding the FP, subjects were required to explore the different targets in the following order: T1, T2, T3 and T4. For each horizontal saccade (black horizontal arrow), the entire set of targets and FP jumped intra-saccadically (short grey arrow). For the pre- and post-adaptation trials of the voluntary saccade experiment, only three targets were presented. When the circle surrounding the FP disappeared, participants were required to make first a vertical saccade towards T1 and then a horizontal saccade towards T2. During the horizontal voluntary saccade, all targets disappeared

saccade (velocity threshold $70-110^{\circ} / \mathrm{sec}$ ) to suppress any visual feedback. These pre-adaptation and post-adaptation blocks were aimed at evaluating the saccadic baseline parameters and the after-effects of adaptation, respectively. Pre-adaptation, adaptation and post-adaptation trials are detailed in the supplementary material.

Data analysis

The beginning and end of primary saccades were detected offline with a custom program developed in Matlab v.7.1 (Mathworks, MA., U.S.A.), using a $50 \%$ sec velocity threshold. For every phase, saccadic gain was calculated as the ratio between saccadic amplitude and initial target eccentricity. Saccades contaminated by a blink and/or associated with an incorrect or absent intra-saccadic visual perturbation, and saccades with gain outside the [mean of 12 saccades \pm 3 standard deviation] range were excluded (in total, $3 \%$ of reactive and $3 \%$ of voluntary saccades in healthy subjects, while 6 and $8 \%$, respectively, in patients). The change of gain of each saccade $(n)$ in the pre-adaptation, adaptation and post-adaptation phases was calculated relative to the pre-adaptation phase as follows:

Gain change of saccade $n$

$$
=\frac{\text { Gain of saccade } n-\text { Mean gain pre }}{\text { Mean gain pre }}
$$


For each subject, a mean gain change was determined for each of the 5 blocks (a25, b25, c25, d40 and e40) of the adaptation phase and for the post-adaptation block ("adaptation after-effect"). Moreover, for each pre-adaptation trial, the duration, latency and peak velocity were computed for all horizontal saccades (i.e., first movement in the reactive saccade task and second movement in the voluntary saccade task). In the case of the voluntary saccade sessions, the termination of the vertical saccade was detected to allow computation of the latency of the horizontal saccade, defined as the duration of the fixation period separating the two saccades. Saccadic gain variability was measured for each subject as the standard deviation of the mean gain in pre-adaptation.

Statistical analyses were performed with the R software (R Development Core Team 2012) using nonparametric tests, as detailed in the supplementary material. Briefly, different statistical tests were used for the pre-adaptation/ post-adaptation phases (Kruskall-Wallis tests) and for the adaptation phase (nonparametric ANOVA). For each of these three phases, the analysis (Kruskall-Wallis or nonparametric ANOVA) allowed to compare patient performances relative to controls, separately for the two saccade types and for the three patients' groups. Significance was set at $p<0.05$.

\section{Results}

Saccadic baseline

The baseline parameters of reactive and voluntary saccades measured in the pre-adaptation phase are presented in Table 2. Each baseline parameter was submitted to Kruskal-Wallis tests (see supplementary material), separately for reactive and voluntary saccades and for the three groups of patients, to assess differences between ipsilesional saccades, contralesional saccades and control saccades (right and left saccades were pooled together for controls). Kruskal-Wallis tests were followed by Dunnett tests, to directly compare controls with patients. For both categories of saccades, the latency did not differ among groups (Kruskal-Wallis tests $<3.3, p>0.19$ ). But, as expected [see e.g. (Alahyane et al. 2007)], the latency was longer for voluntary saccades than for reactive saccades. Compared to controls, the gain of ipsilesional reactive saccades was significantly higher for both SCAAICA patients (Kruskal-Wallis test $=7.7, p<0.05$; Dunnett test, $p<0.001)$ and Wallenberg patients (Kruskal-Wallis test $=4.8, p=0.09$; Dunnett tests, $p<0.05$ ). Regarding contralesional reactive saccades, a higher peak velocity was found in SCA-AICA and Wallenberg patients than in healthy subjects (Kruskal-Wallis tests $=4.8, p=0.09$ and $6.5, p<0.05$, respectively; Dunnett tests, $p<0.001)$. Further, ipsilesional voluntary saccades lasted longer in Wallenberg patients than in controls (Kruskal-Wallis test $=5.5, p=0.06$; Dunnett test, $p<0.001$ ), and this could be linked to the nonsignificant trend of larger saccade gain (Wallenberg: $1.14 \pm 0.35$ versus controls: $0.94 \pm 0.05$ ). PICA patients were more hypometric than controls for contralesional voluntary saccades (Kruskal-Wallis test $=5.5, p=0.06$; Dunnett test, $p<0.001)$ and Wallenberg patients produced contralesional voluntary saccades with higher peak velocity than controls (Kruskal-Wallis test $=5.1, p=0.08$; Dunnett tests, $p<0.001)$. We also compared the intra-subject variability of gain, measured as the standard deviation of the mean, in the pre-adaptation phase between the three groups of patients and the controls, separately for the voluntary and the reactive saccades, but did not find any

Table 2 Baseline parameters of reactive and voluntary saccades

\begin{tabular}{|c|c|c|c|c|c|c|c|}
\hline & \multirow[t]{2}{*}{ Controls } & \multicolumn{2}{|l|}{ PICA patients } & \multicolumn{2}{|c|}{ SCA-AICA patients } & \multicolumn{2}{|l|}{ Wallenberg } \\
\hline & & Ipsi & Contra & Ipsi & Contra & Ipsi & Contra \\
\hline \multicolumn{8}{|c|}{ Reactive saccades } \\
\hline Latency (ms) & $247 \pm 79$ & $238 \pm 54$ & $257 \pm 59$ & $192 \pm 34$ & $208 \pm 59$ & $261 \pm 65$ & $254 \pm 81$ \\
\hline Gain & $0.88 \pm 0.02$ & $0.83 \pm 0.06$ & $0.82 \pm 0.08$ & $0.96 \pm 0.01 * * *$ & $0.94 \pm 0.08$ & $1.03 \pm 0.17 *$ & $0.89 \pm 0.08$ \\
\hline Duration (ms) & $41 \pm 4.3$ & $43 \pm 6.3$ & $43 \pm 7$ & $39 \pm 7.6$ & $40 \pm 7.2$ & $54 \pm 15.9$ & $47 \pm 16.6$ \\
\hline Peak vel. $(\% / s)$ & $243 \pm 19$ & $246 \pm 73$ & $246 \pm 70$ & $310 \pm 67$ & $306 \pm 52 * * *$ & $268 \pm 29$ & $279 \pm 17 * * *$ \\
\hline \multicolumn{8}{|c|}{ Voluntary saccades } \\
\hline Latency (ms) & $438 \pm 157$ & $523 \pm 132$ & $504 \pm 185$ & $570 \pm 120$ & $560 \pm 154$ & $486 \pm 114$ & $513 \pm 199$ \\
\hline Gain & $0.94 \pm 0.05$ & $0.89 \pm 0.04$ & $0.86 \pm 0.05 * * *$ & $0.91 \pm 0.09$ & $0.94 \pm 0.04$ & $1.14 \pm 0.35$ & $0.89 \pm 0.06$ \\
\hline Duration (ms) & $42 \pm 4.2$ & $46 \pm 11.0$ & $49 \pm 16.9$ & $43 \pm 7.1$ & $43 \pm 6.7$ & $63 \pm 22.5^{* * *}$ & $50 \pm 16.6$ \\
\hline Peak vel. $(\% / s)$ & $249 \pm 17$ & $268 \pm 60$ & $249 \pm 67$ & $290 \pm 94$ & $278 \pm 88$ & $269 \pm 70$ & $293 \pm 25 * * *$ \\
\hline
\end{tabular}

Mean values with standard errors of saccade latency, gain, duration and velocity peak (peak vel.)

Significant differences between patients and controls: $* p<0.05$, *** $p<0.001$ (Dunnett tests) 
difference between groups (Kruskal-Wallis tests $<3.4$, $p>0.18)$.

\section{Adaptation time-course}

To estimate the time-course of adaptation induced by the double-step target paradigm, the change in gain for each adaptation trial was computed relative to the mean preadaptation gain. The time-course of adaptive changes induced by the double-step target paradigm is shown in Fig. 2 in one representative subject of each group (control, PICA, SCA-AICA and Wallenberg patients), for reactive saccades (Fig. 2a) and for voluntary saccades (Fig. 2b). Each symbol corresponds to a single saccade. The $x$-axes represent the course of the experimental sessions, and the $y$-axes represent the gain changes calculated relative to the mean gain in pre-adaptation. Saccades in the two directions were plotted separately (all patients represented in this figure had right-sided lesion). As exemplified by the control participant (top row), the intra-saccadic target steps used during the adaptation phase led to a progressive decrease of gain. For this participant, the gain remained reduced in the post-adaptation phase by about $25 \%$ relative to preadaptation for the reactive saccades and by about $11 \%$ for voluntary saccades. A similar adaptation can be observed for the PICA patient (second row). For the SCA-AICA patient (third row), the adaptive changes of the ipsilesional (rightward) voluntary saccades measured at the end of the adaptation phase and in the post-adaptation phase were smaller as compared to the control subject. Finally, for the Wallenberg patient (last row), only small gain changes were observed for the ipsilesional (rightward) reactive and voluntary saccades, during the adaptation phase and hence during the post-adaptation phase as well.

The time-course of adaptation was then studied separately for each group of patients. The change in gain was averaged among participants of each group and plotted as a function of the adaptation block, separately for voluntary and reactive saccades (Fig. 3). A general progressive decrease of gain occurred in all subjects groups, but to a
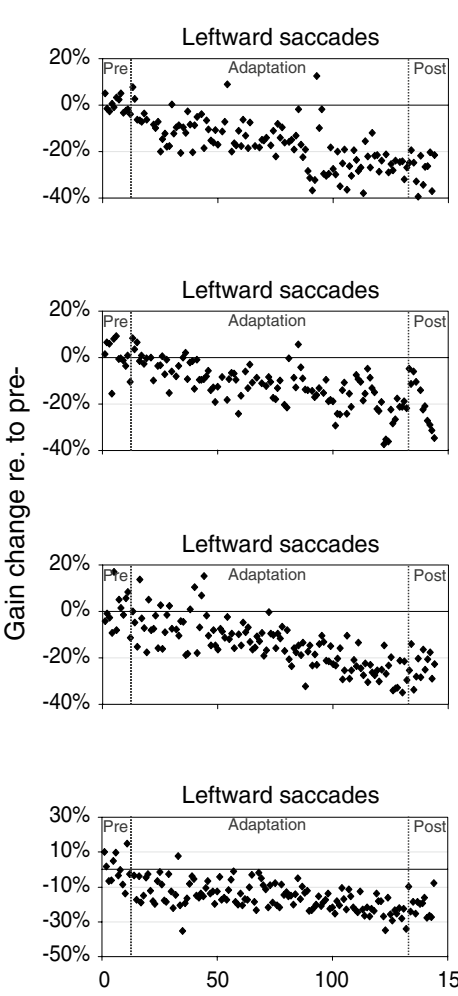

Reactive saccades

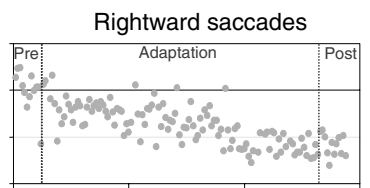

b

Voluntary saccades

\section{Control participant BG}

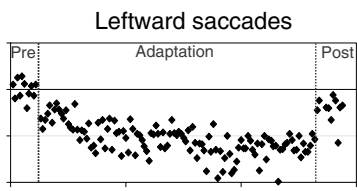

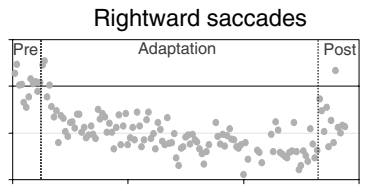

\section{PICA patient $\mathbf{P 1}$}
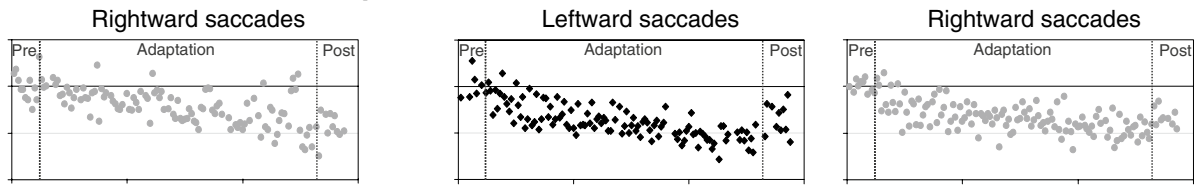

SCA-AICA patient P8
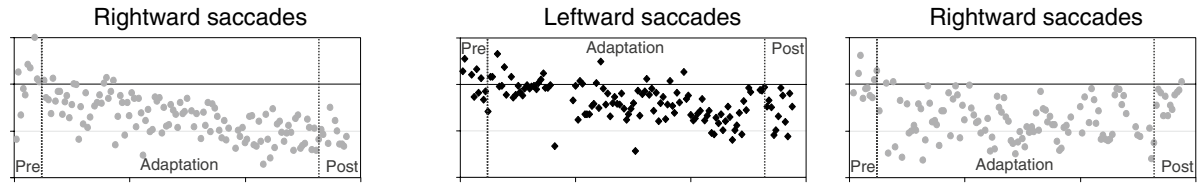

Wallenberg patient P10

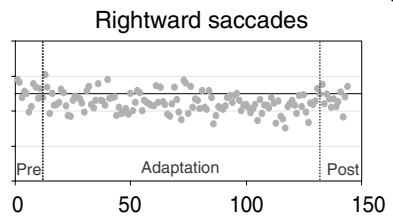

Leftward saccades
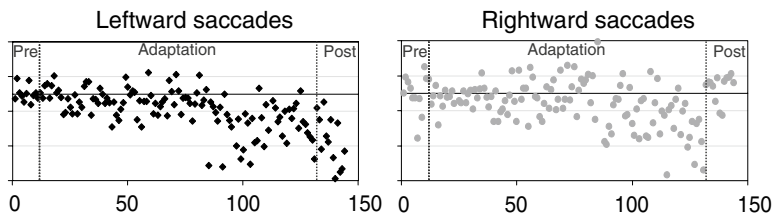

Trial number

Fig. 2 Gain changes of representative subjects of each group for the reactive saccade and the voluntary saccade experiments. The scatter plots represent the trial-by-trial gain changes, relative to mean preadaptation gain, of the reactive saccades (a) and of the voluntary saccades (b). Leftward saccades (black diamonds) and rightward sac- cades (grey discs) are plotted separately. Vertical dotted lines indicate the separation between the different phases: pre-adaptation (Pre), adaptation and post-adaptation (Post). PICA patient P1 and SCAAICA patient P8 had right lesions of the cerebellum. Wallenberg patient $\mathrm{P} 10$ had a right lesion of the lateral medulla 
Adaptation time-course

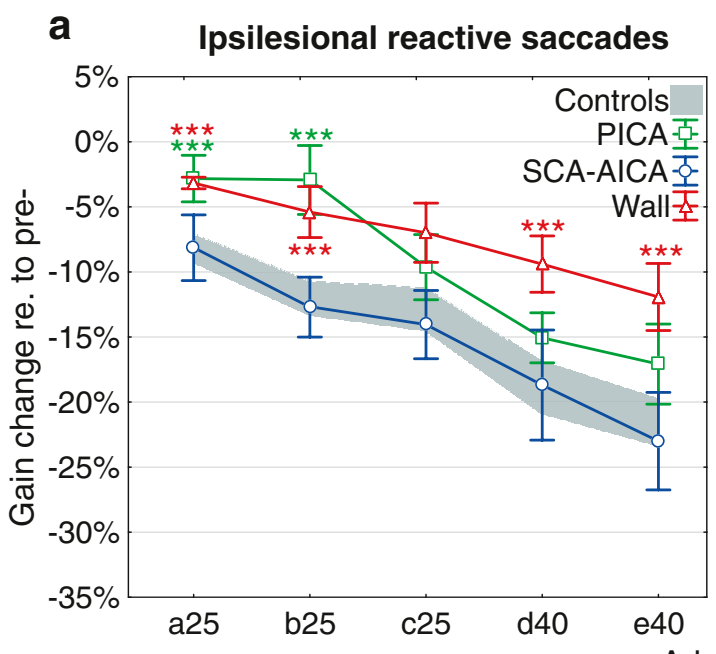

b Contralesional reactive saccades

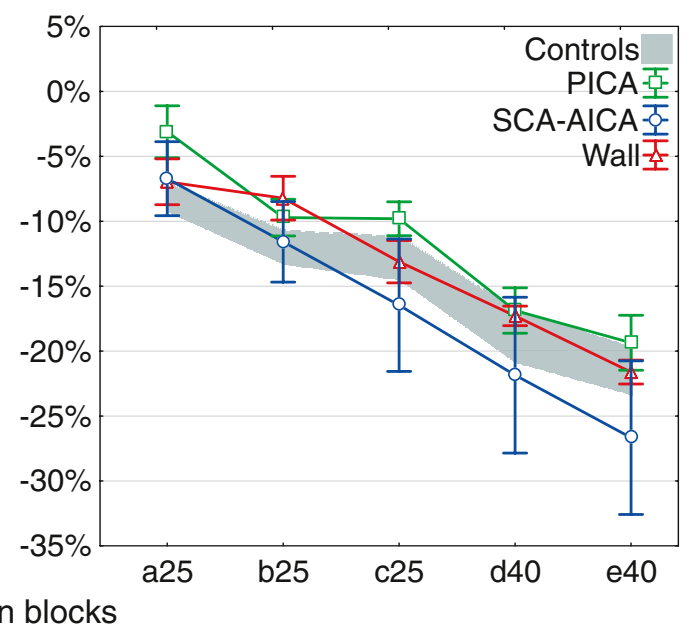

\section{C}

Ipsilesional voluntary saccades

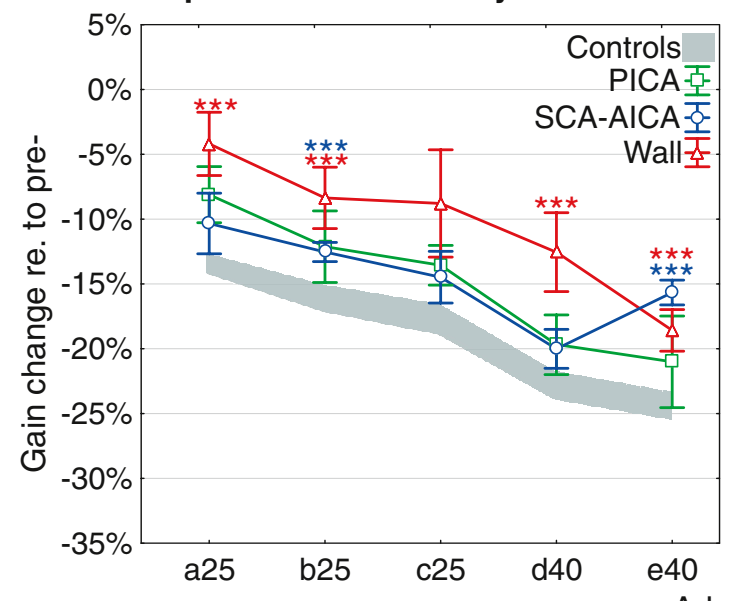

d
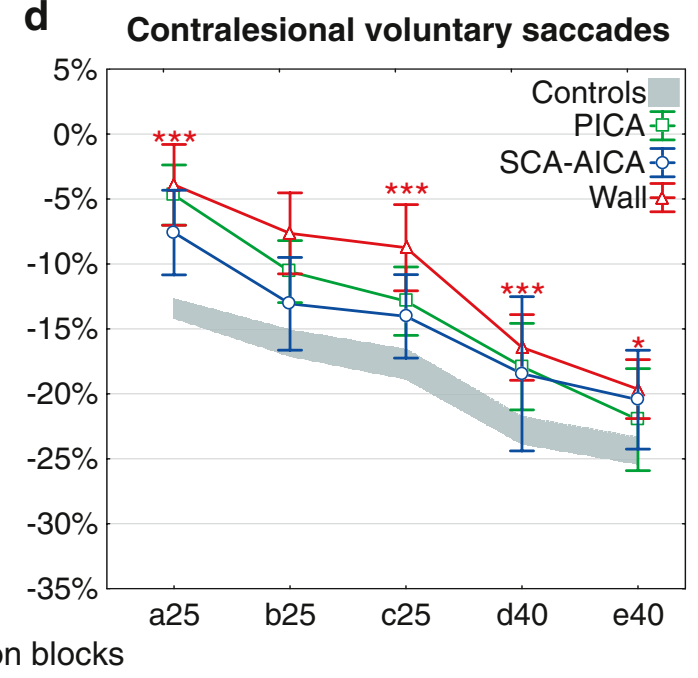

Fig. 3 Development of the adaptation for the different groups of patients and for the controls. Gain changes relative to the mean gain in pre-adaptation are plotted as a function of adaptation blocks: a25, b25, c25 (perturbation of visual stimulus of $25 \%$ of initial target eccentricity) and c40, d40 (perturbation of $40 \%$ ). These plots were built separately for reactive (a, b) and voluntary (c, d) saccades produced in the ipsilesional $(\mathbf{a}, \mathbf{c})$ and contralesional $(\mathbf{b}, \mathbf{d})$ directions. The grey-shaded areas represent mean values \pm 1 standard error of the mean (SEM) of gain changes measured for the control group (data of rightward and leftward saccades were averaged). The green, blue and red lines and symbols correspond to gain changes, respectively, for the PICA, SCA-AICA and Wallenberg patients (error bars show SEMs). Asterisks (same colour code) indicate significant differences of gain changes for a particular adaptation block between PICA, SCA-AICA or Wallenberg patients and the control group: * $p<0.05$, $* * * p<0.001$ (Dunnett tests)

the "adaptation blocks" factor in all six ANOVAs (nonparametric ANOVA, $p<0.001)$. However, differences between controls and patients were also revealed with these nonparametric ANOVAs. For the PICA patients, a main effect of the group factor on gain changes was found for reactive saccades $(p<0.05)$, without any interaction with the adaptation blocks $(p=0.34)$. This main effect was related to the fact that, as compared to control subjects, a smaller decrease in gain for ipsilesional saccades during the first two adaptation blocks could be observed (Fig. 3a-Dunnett ing the adaption phase was confirmed by the main effect of 
tests, $p<0.001)$. No difference between PICA patients and controls was detected for the adaptive changes of voluntary saccades (Fig. 3c, d-nonparametric ANOVA, group factor and group $\times$ adaptation blocks interaction, $p>0.6$ ). In the case of SCA-AICA patients, gain changes for reactive saccades were similar to the ones of the control participants (Fig. 3a, b-nonparametric ANOVA, group factor and group $\times$ adaptation blocks interaction, $p>0.13$ ). However, a significant interaction between the group factor and the block factor was revealed for the voluntary saccades $(p<0.05)$. Indeed, ipsilesional voluntary saccades were less shortened for the patients in the third adaptation block and in the last adaptation block than for the control subjects (Dunnett tests, $p<0.001$ ). Surprisingly, the difference between controls and SCA-AICA patients in the last adaptation block was due to a quick drop of adaptation for the patients (Fig. 3c). This drop was specific to the ipsilesional, but not to the contralesional saccades. Note, however, that, although present in all 4 patients of this group, the mean effect is mainly driven by patient P6 who showed a much larger drop of adaptation between the fourth and last adaptation blocks (about $10 \%$ ) than the other three patients (only 2-3\%, data not shown). Finally, Wallenberg patients showed strong deficits in adaptation time-course for both reactive and voluntary saccades (nonparametric ANOVA, group effect, $p<0.001$ and $p<0.01$, respectively). Indeed, gain changes of ipsilesional reactive saccades were smaller for Wallenberg patients than for controls for most of the adaptation phase, except the third block (Fig. 3a-Dunnett tests, $p<0.001$ ). Similarly, the adaptive changes for both directions of voluntary saccades were strongly impaired for the patients compared to controls (see Fig. 3c, d, Dunnett tests, $p<0.05$ ).

To summarise, for PICA patients, the decrease in ipsilesional reactive saccade gain was smaller than in healthy subjects only at the beginning of the adaptation phase. For SCA-AICA patients, a reduced gain change relative to controls was observed for ipsilesional voluntary saccades, at the very end of the adaptation phase, but this effect was mainly driven by one patient. Finally, Wallenberg patients showed a poor decrease in gain for ipsilesional reactive saccades and for both voluntary saccades directions for most of the adaptation phase.

\section{Adaptation after-effect}

The change in saccade gain in the post-adaptation phase when the visual perturbation was stopped provided a measure of adaptation after-effect. It is therefore considered to specifically assess the amount of adaptation, independently of any cognitive or strategic factors (Bastian 2008). As shown in Fig. 4, after-effects differed among groups. Kruskal-Wallis tests (see supplementary material) were performed separately for reactive and voluntary saccades and for the three groups of patients, to compare aftereffects between controls and the ipsilesional and contralesional saccades of the patients. PICA patients had similar after-effects than controls for both saccade types (Fig. 4a, $\mathrm{b}$-Kruskal-Wallis test $<1.8, p>0.40$ ). The after-effects of reactive saccades were also similar for SCA-AICA patients and controls (Fig. 4b-Kruskal-Wallis test $=0.38$, $p=0.83$ ). However, the after-effect of ipsilesional voluntary saccades was significantly smaller for SCA-AICA patients compared to control subjects (Kruskal-Wallis test $=5.1, p=0.08$; Dunnett tests, $p<0.001$ ). Because the reduced gain change at the end of the adaptation phase was mainly driven by one SCA-AICA patient (see previous section), we looked at the after-effect separately in each patient. The after-effects of the patients ranged from -4 to $-12 \%$ (mean $-8.1 \pm 3.1 \%$ ), whereas the after-effects of the 6 control participants ranged from -10 to $-20 \%$ (mean $-15.4 \pm 3.9 \%$ ). Thus, a reduced after-effect for ipsilesional voluntary saccades appears consistent for all SCAAICA patients. Concerning Wallenberg patients, a strong reduction of after-effect was observed for both ipsilesional and contralesional reactive saccades (Fig. 4a-KruskalWallis test $=8.8, p<0.05$; Dunnett tests, $p<0.001$ and $p<0.05$, respectively) and for ipsilesional voluntary saccades (Fig. 4b-Kruskal-Wallis test $=4.5, p=0.10$; Dunnett tests, $p<0.05$ ).

Thus, the adaptation after-effect was significantly and consistently reduced, relative to controls, in SCA-AICA patients for ipsilesional voluntary saccades, in Wallenberg patients for reactive saccades bidirectionally and again in Wallenberg patients for ipsilesional voluntary saccades.

\section{Discussion}

The main aim of this study was to investigate the role of different cerebellar territories in saccadic adaptation. Before discussing this aspect, we compare the baseline saccade parameters between the different subjects groups.

Saccade metrics and cerebellar lesions

One of the typical features of Wallenberg syndrome is the saccadic lateropulsion, characterised by ipsilesional saccades hypermetria, contralesional saccades hypometria and ipsilesional deviation of vertical saccades (Helmchen et al. 1994; Straube et al. 1994; Tilikete et al. 2006). As indicated in "Introduction", saccadic lateropulsion could reflect a dysfunction of the fastigial nucleus, resulting from damage of the afferent pathway from the inferior olive. In our Wallenberg patients, the ipsilesional hypermetria tendency is consistent with saccadic lateropulsion, and with a damage 


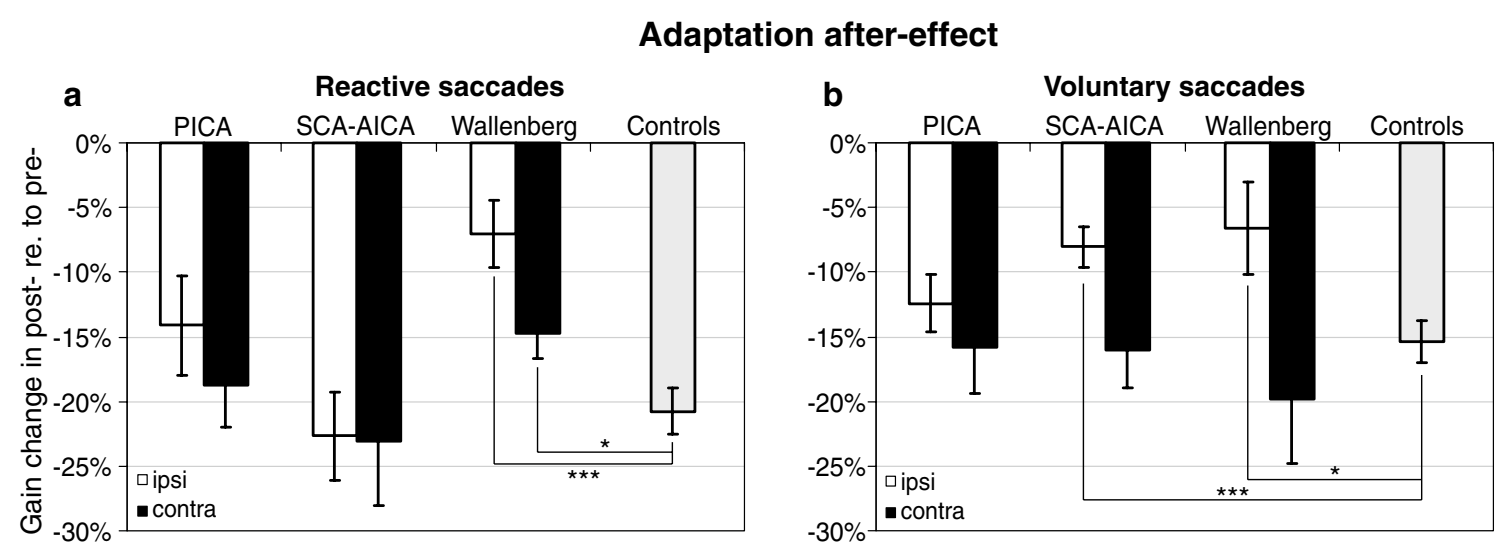

Fig. 4 Adaptation after-effects for reactive saccades (a) and for voluntary saccades (b). After-effects were calculated as gain changes in post-adaptation relative to the mean gain measured in pre-adaptation. Mean values are plotted separately for the ipsilesional saccades (white bars) and for the contralesional saccades (black bars)

of this pathway, despite the lack of contralesional hypometria. The absence of hypometria might reflect an incomplete clinical picture and/or insufficient evaluation (target eccentricity too small). The fact that the hypermetria tendency was observed for both reactive and voluntary saccades further suggests that the olivo-cerebellar pathway could be implicated in the control of these two saccade categories. This hypothesis is consistent with data in monkeys, which showed that the oculomotor vermis contributes similarly to the production of reactive and scanning voluntary saccades (Kojima et al. 2010). This hypothesis is also supported by recent fMRI data in humans (Schraa-Tam et al. 2009).

Our PICA and SCA-AICA patients showed hypometric contralesional voluntary saccades and hypermetric ipsilesional reactive saccades, respectively. As mentioned in "Methods", the lesion predominantly affected the cerebellar hemispheres in both groups of patients. This suggests that the control of saccade metrics is also implemented in cerebellar territories other than the vermis. Some studies have reached this conclusion for reactive saccades (Dieterich et al. 2000; Hayakawa et al. 2002; Panouilleres et al. 2012a; Schraa-Tam et al. 2009). Two fMRI studies also suggested that nonvermal cerebellar regions, lobules VI and Crus I, could play a role in the control of reactive and voluntary saccades (Hayakawa et al. 2002; SchraaTam et al. 2009). These hemispheric lobules are located above the horizontal fissure, in the vascular domain of the SCA (Schmahmann et al. 2000; Tatu et al. 1996). In complement with these findings, our results suggest that the supero-anterior cerebellum is implicated in the control of reactive saccades metrics and that the infero-posterior cerebellum (vascular domain of the PICA) would play a more important role in the control of voluntary saccade metrics. of the three groups of patients and as the average of the rightward and leftward saccades of the control group (grey bars). Error bars show SEMs. Asterisks indicate significant differences of after-effects between PICA, SCA-AICA or Wallenberg patients and the control group: $* p<0.05, * * * p<0.001$ (Dunnett tests)

\section{Adaptation of reactive saccades and cerebellar lesions}

A crucial contribution of the cerebellum to reactive saccades adaptation has been repeatedly demonstrated (reviewed in Hopp and Fuchs 2004; Pelisson et al. 2010; Prsa and Thier 2011; Tian et al. 2009). Lesions studies in monkeys showed the involvement of medio-posterior cerebellum, composed of vermal lobules VI-VII and of the caudal part of the fastigial nucleus, in saccadic plasticity (Barash et al. 1999; Iwamoto and Kaku 2010; Optican and Robinson 1980; Takagi et al. 1998). Similarly, lesion studies in human patients consistently point to the key role of the cerebellum in reactive saccade adaptation (Alahyane et al. 2008a; Choi et al. 2008; Golla et al. 2008; Straube et al. 2001; Waespe and Baumgartner 1992). Golla et al. (2008) specifically reported that only the patients with lesion including the vermis showed impaired adaptation. Moreover, Xu-Wilson et al. (2009) showed that patients with spinocerebellar ataxia type 6 (SCA6), a neurodegenerative disease mainly affecting the Purkinje cells and especially the vermis, presented strong deficits of saccadic adaptation. In comparison, the effects of lateral medullary infarctions on saccadic adaptation are still discussed, as decreased capabilities of adaptation in Wallenberg patients were reported in one study (Waespe and Baumgartner 1992), but not in the other (Choi et al. 2008). Our own results disclosed a strong deficit of saccadic adaptation in Wallenberg patients. Note that contrary to the present study and to Waespe and Baumgartner's (1992), patients in Choi et al.'s study did not present clear signs of saccadic lateropulsion. This last observation suggests that the olivocerebellar saccadic pathway was partly spared in their Wallenberg patients, which could account for their normal saccadic adaptation capabilities. 
Medio-posterior cerebellum and error signal for saccadic adaptation

We found that Wallenberg patients presented strong impairment of ipsilesional saccades adaptation and characterised by slow development and weak after-effect of adaptive changes. The slowing of adaptation time-course seems to suggest that the olivo-cerebellar pathway is involved in the transmission of a signal necessary for the establishment of adaptive changes. Recent studies in monkeys proposed that an error signal leading to saccadic adaptation is produced at the level of the deep layers of the superior colliculus (Kaku et al. 2009; Soetedjo et al. 2009). Kaku et al. (2009) further proposed that this signal would be conveyed to the oculomotor vermis via the inferior olivary nucleus. Our data in Wallenberg patients are compatible with this hypothesis, and because adaptation of both reactive and voluntary saccades was impaired in these patients, our study supports the hypothesis that this collicular-olivo-cerebellar pathway can be implicated in conveying adaptive error signals for both saccade categories. Alternatively, our results are also compatible with the popular forward model hypothesis (Wolpert et al. 1998). According to this hypothesis, the cerebellum uses an efference copy of motor commands to compute a prediction of visual feedback and ultimately an error signal required for sensorimotor adaptation. Saccadic adaptation deficits in our Wallenberg patients could then result from an alteration of error signal related to an impaired cerebellar forward model. We therefore conclude that the medio-posterior cerebellum plays a critical role in the adaptation of the two saccade types, either in modifying the oculomotor commands by processing an error signal transmitted through the olivo-cerebellar pathway or in computing this error signal necessary for adaptation.

Hemispheric cerebellar structures involved in saccadic adaptation

Postero-inferior cerebellar artery patients presented essentially an abnormally slow decrease in ipsilesional reactive saccades gain at the beginning of the adaptation phase. However, gain changes that occurred later during the adaptation phase and the after-effects measured in post-adaptation matched those of controls. These partial deficits suggest that the territories vascularised by PICA are not really critical for saccadic adaptation. On the contrary, SCAAICA patients presented, for ipsilesional voluntary saccades, a consistently smaller gain decrease than controls in post-adaptation, and a less consistent, albeit significant, difference with controls in the last adaptation block. This suggests that the damaged cerebellar structures are involved in late acquisition and/or maintenance of voluntary saccades adaptation. A recent study on the adaptation of the upper limb to visuomotor rotation concluded that SCA patients show impairment of both error reduction and after-effect of adaptation, whereas PICA patients were mainly impaired during the error reduction phase (Werner et al. 2010). Thus, for both the voluntary movements of the upper limb and of the eyes, the superior part of the cerebellum could be crucial for the after-effect of sensorimotor adaptation.

In conclusion, this study shows for the first time that the modulation of the medio-posterior cerebellum by the olivocerebellar pathway contributes to the development of adaptation of both reactive and voluntary saccades. Our results also highlight that the supero-anterior part of the cerebellar hemispheres is specifically involved in the adaptation of voluntary saccades.

Acknowledgments This work was performed in the "Mouvement et Handicap" platform (Lyon, France), approved by the local ethic committee (CCPPRB-Lyon B) and supported by "Hospices Civils de Lyon" (HCL/P 2002.303) and by the French National Research Agency (Grant ANR-06-NEURO-001). We thank all subjects and patients for their kind participation. This work was performed within the framework of the LABEX CORTEX (ANR-11-LABX-0042) of Université de Lyon, within the program "Investissements d'Avenir" (ANR-11-IDEX-0007) operated by the French National Research Agency (ANR).

\section{References}

Alahyane N, Pelisson D (2005) Retention of saccadic adaptation in humans. Ann N Y Acad Sci 1039:558-562

Alahyane N, Salemme R, Urquizar C, Cotti J, Guillaume A, Vercher JL, Pelisson D (2007) Oculomotor plasticity: are mechanisms of adaptation for reactive and voluntary saccades separate? Brain Res 1135:107-121

Alahyane N, Devauchelle AD, Salemme R, Pelisson D (2008a) Spatial transfer of adaptation of scanning voluntary saccades in humans. NeuroReport 19:37-41

Alahyane N, Fonteille V, Urquizar C, Salemme R, Nighoghossian N, Pelisson D, Tilikete C (2008b) Separate neural substrates in the human cerebellum for sensory-motor adaptation of reactive and of scanning voluntary saccades. Cerebellum 7:595-601

Barash S, Melikyan A, Sivakov A, Zhang M, Glickstein M, Thier P (1999) Saccadic dysmetria and adaptation after lesions of the cerebellar cortex. J Neurosci 19:10931-10939

Bastian AJ (2008) Understanding sensorimotor adaptation and learning for rehabilitation. Curr Opin Neurol 21:628-633

Choi KD, Kim HJ, Cho BM, Kim JS (2008) Saccadic adaptation in lateral medullary and cerebellar infarction. Exp Brain Res 188:475-482

Collins T, Dore-Mazars K (2006) Eye movement signals influence perception: evidence from the adaptation of reactive and volitional saccades. Vis Res 46:3659-3673

Cotti J, Guillaume A, Alahyane N, Pelisson D, Vercher JL (2007) Adaptation of voluntary saccades, but not of reactive saccades, transfers to hand pointing movements. J Neurophysiol 98:602-612

Cotti J, Panouilleres M, Munoz DP, Vercher JL, Pelisson D, Guillaume A (2009) Adaptation of reactive and voluntary saccades: different patterns of adaptation revealed in the antisaccade task. J Physiol 587:127-138 
Desmurget M, Pelisson D, Urquizar C, Prablanc C, Alexander GE, Grafton ST (1998) Functional anatomy of saccadic adaptation in humans. Nat Neurosci 1:524-528

Deubel H (1995) Separate adaptive mechanisms for the control of reactive and volitional saccadic eye movements. V Res 35:3529-3540

Dieterich M, Bucher SF, Seelos KC, Brandt T (2000) Cerebellar activation during optokinetic stimulation and saccades. Neurology 54:148-155

Erkelens CJ, Hulleman J (1993) Selective adaptation of internally triggered saccades made to visual targets. Exp Brain Res 93:157-164

Ethier V, Zee DS, Shadmehr R (2008) Spontaneous recovery of motor memory during saccade adaptation. J Neurophysiol 99: $2577-2583$

Fujita M, Amagai A, Minakawa F, Aoki M (2002) Selective and delay adaptation of human saccades. Brain Res Cogn Brain Res 13:41-52

Gerardin P, Miquee A, Urquizar C, Pelisson D (2012) Functional activation of the cerebral cortex related to sensorimotor adaptation of reactive and voluntary saccades. NeuroImage 61:1100-1112

Glickstein M, Sultan F, Voogd J (2011) Functional localization in the cerebellum. Cortex 47:59-80

Golla H, Tziridis K, Haarmeier T, Catz N, Barash S, Thier P (2008) Reduced saccadic resilience and impaired saccadic adaptation due to cerebellar disease. Eur J Neurosci 27:132-144

Hayakawa Y, Nakajima T, Takagi M, Fukuhara N, Abe H (2002) Human cerebellar activation in relation to saccadic eye movements: a functional magnetic resonance imaging study. Ophthalmologica 216:399-405

Helmchen C, Straube A, Buttner U (1994) Saccadic lateropulsion in Wallenberg's syndrome may be caused by a functional lesion of the fastigial nucleus. J Neurol 241:421-426

Hopp JJ, Fuchs AF (2004) The characteristics and neuronal substrate of saccadic eye movement plasticity. Prog Neurobiol 72:27-53

Iwamoto Y, Kaku Y (2010) Saccade adaptation as a model of learning in voluntary movements. Exp Brain Res 204:145-162

Jenkinson N, Miall RC (2010) Disruption of saccadic adaptation with repetitive transcranial magnetic stimulation of the posterior cerebellum in humans. Cerebellum 9(4):548-555

Johnston K, Everling S (2008) Neurophysiology and neuroanatomy of reflexive and voluntary saccades in non-human primates. Brain Cogn 68:271-283

Kaku Y, Yoshida K, Iwamoto Y (2009) Learning signals from the superior colliculus for adaptation of saccadic eye movements in the monkey. J Neurosci 29:5266-5275

Kojima Y, Soetedjo R, Fuchs AF (2010) Behavior of the oculomotor vermis for five different types of saccade. J Neurophysiol 104:3667-3676

McLaughlin SC (1967) Parametric adjustment in saccadic eye movements. Percept Psychophys 2:359-362

Muri R, Nyffeler T (2008) Neurophysiology and neuroanatomy of reflexive and volitional saccades as revealed by lesion studies with neurological patients and transcranial magnetic stimulation (TMS). Brain Cogn 68:284-292

Optican LM, Robinson DA (1980) Cerebellar-dependent adaptive control of primate saccadic system. J Neurophysiol 44:1058-1076

Panouilleres M, Urquizar C, Salemme R, Pelisson D (2011) Sensory processing of motor inaccuracy depends on previously performed movement and on subsequent motor corrections: a study of the saccadic system. PLoS ONE 6:e17329

Panouilleres M, Neggers SFW, Gutteling TP, Salemme R, van der Stigchel S, van der Geest JN, Frens MA, Pelisson D (2012a) Transcranial magnetic stimulation and motor plasticity in Human lateral cerebellum: dual effect on saccadic adaptation. Hum Brain Mapp 33:1512-1525
Panouilleres M, Salemme R, Urquizar C, Pelisson D (2012b) Effect of saccadic adaptation on sequences of saccades. J Eye Mov Res $5: 1-13$

Panouilleres M, Gaveau V, Socasau C, Urquizar C, Pelisson D (2013) Brain processing of visual information during fast eye movements maintains motor performance. PLoS ONE 8:e54641

Pelisson D, Alahyane N, Panouilleres M, Tilikete C (2010) Sensorimotor adaptation of saccadic eye movements. Neurosci Biobehav Rev 34:1103-1120

Prsa M, Thier P (2011) The role of the cerebellum in saccadic adaptation as a window into neural mechanisms of motor learning. Eur $\mathrm{J}$ Neurosci 33:2114-2128

R Development Core Team (2012) R: a language and environment for statistical computing. R Foundation for Statistical Computing, Vienna

Schmahmann JD, Doyon J, Toga AW, Evans AC (2000) MRI atlas of the human cerebellum. Academic Press, San Diego

Schmahmann JD, Gardner R, MacMore J, Vangel MG (2009) Development of a brief ataxia rating scale (BARS) based on a modified form of the ICARS. Mov Dis 24:1820-1828

Schraa-Tam CK, van Broekhoven P, van der Geest JN, Frens MA, Smits M, van der Lugt A (2009) Cortical and cerebellar activation induced by reflexive and voluntary saccades. Exp Brain Res 192:175-187

Soetedjo R, Fuchs AF, Kojima Y (2009) Subthreshold activation of the superior colliculus drives saccade motor learning. J Neurosci 29:15213-15222

Straube A, Helmchen C, Robinson F, Fuchs A, Buttner U (1994) Saccadic dysmetria is similar in patients with a lateral medullary lesion and in monkeys with a lesion of the deep cerebellar nucleus. J Vestib Res 4:327-333

Straube A, Deubel H, Ditterich J, Eggert T (2001) Cerebellar lesions impair rapid saccade amplitude adaptation. Neurology 57:2105-2108

Takagi M, Zee DS, Tamargo RJ (1998) Effects of lesions of the oculomotor vermis on eye movements in primate: saccades. J Neurophysiol 80:1911-1931

Tatu L, Moulin T, Bogousslavsky J, Duvernoy H (1996) Arterial territories of human brain: brainstem and cerebellum. Neurology 47:1125-1135

Tian J, Ethier V, Shadmehr R, Fujita M, Zee DS (2009) Some perspectives on saccade adaptation. Ann N Y Acad Sci 1164:166-172

Tilikete C, Koene A, Nighoghossian N, Vighetto A, Pelisson D (2006) Saccadic lateropulsion in Wallenberg syndrome: a window to access cerebellar control of saccades? Exp Brain Res 174:555-565

Waespe W, Baumgartner R (1992) Enduring dysmetria and impaired gain adaptivity of saccadic eye movements in Wallenberg's lateral medullary syndrome. Brain 115(Pt 4):1123-1146

Werner S, Bock O, Gizewski ER, Schoch B, Timmann D (2010) Visuomotor adaptive improvement and after effects are impaired differentially following cerebellar lesions in SCA and PICA territory. Exp Brain Res 201:429-439

Wolpert DM, Miall RC, Kawato M (1998) Internal models in the cerebellum. Trends Cong Sci 2:338-347

Wong AL, Shelhamer M (2011) Sensorimotor adaptation error signals are derived from realistic predictions of movement outcomes. J Neurophysiol 105:1130-1140

Xu-Wilson M, Chen-Harris H, Zee DS, Shadmehr R (2009) Cerebellar contributions to adaptive control of saccades in humans. J Neurosci 29:12930-12939

Zimmermann E, Lappe M (2009) Mislocalization of flashed and stationary visual stimuli after adaptation of reactive and scanning saccades. J Neurosci 29:11055-11064 